\title{
Maternal Karyogene and Cytoplasmic Genotype Affect the Induction Efficiency of Doubled Haploid Inducer in Brassica napus
}

\section{Wei Zhang}

Institute of Crop Research, Chengdu Academy of Agricultural and Forestry Sciences

YongTing $\mathrm{Ma}$

Institute of Crop Research, Chengdu Academy of Agricultural and Forestry Sciences

\section{ZhenDong Zhu}

Institute of Crop Research, Chengdu Academy of Agricultural and Forestry Sciences

\section{LiangJun Huang}

Institute of Crop Research, Chengdu Academy of Agricultural and Forestry Sciences

\section{Asif Ali}

Rice Research Institute, Sichuan Agricultural University

\section{Xuan Luo}

Maize Research Institute, Sichuan Agricultural University

\section{Ying Zhou}

Institute of Crop Research, Chengdu Academy of Agricultural and Forestry Sciences

Yun Li

Institute of Crop Research, Chengdu Academy of Agricultural and Forestry Sciences

\section{Peizhou Xu}

Rice Research Institute, Sichuan Agricultural University

\section{Jin Yang}

Institute of Crop Research, Chengdu Academy of Agricultural and Forestry Sciences

\section{Zhuang Li}

Agricultural College, Sichuan Agricultural University

\section{HaoRan Shi}

Institute of Crop Research, Chengdu Academy of Agricultural and Forestry Sciences Jisheng Wang

Institute of Crop Research, Chengdu Academy of Agricultural and Forestry Sciences

\section{WanZhuo Gong}

Institute of Crop Research, Chengdu Academy of Agricultural and Forestry Sciences

\section{Qiong Zou}

Institute of Crop Research, Chengdu Academy of Agricultural and Forestry Sciences

\section{LanRong Tao}

Institute of Crop Research, Chengdu Academy of Agricultural and Forestry Sciences 
Institute of Crop Research, Chengdu Academy of Agricultural and Forestry Sciences

\section{Rong Tang}

Institute of Crop Research, Chengdu Academy of Agricultural and Forestry Sciences

\section{ZhangJie Zhao}

Agricultural College, Sichuan Agricultural University

\section{Zhi Li}

Agricultural College, Sichuan Agricultural University

\section{ShiXing Guo}

Agricultural College, Sichuan Agricultural University

\section{Shaohong Fu ( $\square$ honzon2010@163.com )}

Institute of Crop Research, Chengdu Academy of Agricultural and Forestry Sciences

\section{Research Article}

Keywords: double haploid inducer, paternal infiltration, chromosome elimination, polyploid, Brassica napus

Posted Date: February 19th, 2021

DOI: https://doi.org/10.21203/rs.3.rs-210233/v1

License: (c) (i) This work is licensed under a Creative Commons Attribution 4.0 International License. Read Full License 


\section{Abstract \\ Background}

Production of pure lines by double haploid induction provides a new way to achieve homozygosity earlier in Brassica napus. Previously, it is not known, whether the induction has obvious maternal genotypic differences.

\section{Results}

In this study, different karyogene and cytoplasmic genotype of Brassica napus was pollinated with the inducers Y3380 and Y3560. Using the identification of the ploidy, fertility and SSR analysis of the induced F1 generation, it was found that the ploidy and phenotype of the induced F1 plants were consistent with maternal parent. The SNP chip analysis revealed that induction efficiency was affected by the karyogene when the maternal cytoplasmic genotype was the same. However, this efficiency was affected by the cytoplasmic genotype when the karyogenes were same, and the offspring of the ogura cytoplasm easier to high-frequency inducer gene hybridization or low-frequency infiltration.

\section{Conclusion}

The induction effect is influenced by the interaction between maternal karyogene and cytoplasmic genotype, and the results from the partial hybridization of progeny chromosomes indicate that the induction process may be attributed to the selective elimination of paternal chromosome. This study provides a basis for exploring the mechanism of DH inducer in Brassica napus, and provides new insights for utilization of inducers inbreeding.

\section{Background}

Brassica napus (AACC, $2 n=4 x=38$ ) is an allotetraploid plant derived from Brassica rape $(A A, 2 n=20)$ and Brassica oleracea $(C C, 2 n=18)$ through interspecific cross and natural doubling of chromosomes that happened about 75 million years ago [1, 2]. Breeding of Brassica napus varieties has appreciated the utilization of heterosis that mainly includes different technical methods e.g. polima cytoplasmic sterility (pol $\mathrm{CMS}$ ) and ogura cytoplasmic sterility (ogu CMS). Before the advent of successful application of microspore culture in Brassica napus, the pure lines were used to obtained by means of multi-generational selfing [3-5], breeding cycle was long. Isolated microspore culture is usually influenced by maternal genotype, environment, temperature and many other abiotic factors [6-8]. Rapeseed scientists are keen to find that is there a simpler and more efficient technique than isolated microspore culture that can quickly and efficiently obtain pure lines of Brassica napus? In recent years, in-vivo haploid induction line was derived from the maize Stock6 [9] and the haploid induction line mediated by the Arabidopsis CENH3 gene have been successfully used in maize [10], barley [11], and rice [12]. Maize haploid induction gene ZmPLA1 [13] and Arabidopsis gene CENH3 [1416] have been applied to wheat, and haploid induction have been achieved. In addition, the use of barley bulb method and distant pollination of corn pollens in wheat have also achieved haploid induction, in which induction rate was about $20-45 \%$ [14]. It is easier to get pure lines induced by crossing with haploids than 
isolated microspore culture. A recent study reported, in which an allotetraploid Brassica napus was crossed with an allo-octaploid rape (AAAACCCC, $2 n=8 x \approx 76$ ) [17] and two allo-octaploid rapes had induced the function of the maternal parent to produce double haploids $(\mathrm{DH})$ and named as the $\mathrm{DH}$ induction lines in Brassica napus: Y3560 and Y3380 [18]. SSR molecular markers, plant ploidy, and morphological identification revealed that a higher proportion of plants in the F1 generation were similar to the maternal parent and induction efficiency ranges from 34.09\% 98.66\%. What accounts for such a huge difference in induction efficiency? And it was observed that there were different induction effects according to maternal cytoplasmic genotypes of Brassica napus. Whether the induction efficiency was related to the cytoplasmic genotype of the maternal parent? Therefore, in this study we used DH inducer lines as paternal parent to pollinate three types of pol, ogu and nap cytoplasmic maternal parents, and ploidy, phenotype and genotypes of the induced offspring were observed. SNP analysis was performed to evaluate the relationship of maternal parent cytoplasmic effect. This study lays the foundation for the application of the DH lines in Brassica napus and contribute to the understanding of maternal karyogenic and cytoplasmic genetypic effects.

\section{Results}

\section{Ploidy analysis}

The offspring of a hybrid between high-ploidy and low-ploidy that are prone to intermediate ploidy [19]. In order to understand whether there were differences in ploidy level before and after induction, we selected different Brassica napus induced F1, and tetraploid Brassica napus ZS11, which was used as control. The detection results are as follows (Fig 1, Additional file 1, Additional file 2). The fluorescence intensity of F1 generation obtained by pollination of $Y 3560$ and four maternal parents was about 409.5 510.5D thousand lines. The fluorescence intensity of F1 generation of Y3380 pollinated with five maternal parents was about 398.9 521.1D thousand lines, and the detection results were almost the same as peak value of 423.1 487.7D thousand lines in control hybrid offsprings, so they were all tetraploid plants, too. At the same time, we tested the ploidy of pollinated maternal parents and DH inducer. The fluorescence intensity of the maternal parent plants ranged from 406.9 502.1D thousand lines, all of which were tetraploid plants. The fluorescence intensity of inducer was about 753.2 852.5D thousand lines, which was two times ZS11 as control, so all of them were octoploid plants. However, in this study we selected octoploid rapeseed DH lines as the paternal parent of the tetraploid Brassica napus. The offspring ploidy were consistent their maternal parent ploidy (tetraploid), the preliminary explanation of octoploid and tetraploid rape crossing does not make sense but the tetraploid Bassica napus were pollinated by octoploid rape, which has played an induced or partial chromosomal hybrid effect.

\section{Plant morphology and fertility investigation}

Fertility and plant morphology investigations (Fig 2, Additional file 3) of F1 showed that most of plant were steriles, while only a few were fertile. Which sterile lines were pollinated by the DH inducer lines (used as paternal parent), while those of the fertile plants have shown no obvious difference from the maternal parents except for the flower morphology. Fertility identification results of paternal parent Y3560, Y3380 and induced F1 were shown as follows Additional file 3. The homozygous and stable pol CMS of 0068A, 0933A, and D717A that were used as the maternal parent crossed with Y3560 and six, two, and one fertile plant were 
appeared in their offspring, respectively. Only one fertile plant was appeared in F1, when Y3380 was used as the paternal parent and homozygous pol CMS 0933A was used as maternal parent; fertile plants were not found in F1, when Y3380 was crossed with L0068A and L0933A as maternal parent. At same time, the hybrid progeny ZS11 was also used as paternal parent for crossing with different maternal parents, and all resulting progeny produced were semi-sterile or sterile plants. Results of fertility identification revealed that among the induced offspring from paternal parents $Y 3560$ and $Y 3380$, the fertile plants appeared in the offspring of pol CMS, when it was used as maternal parent with a probability of $2.22 \%$ to $30.00 \%$. Among them, the probability of offspring (0068A×Y3560) was highest $(30.00 \%)$ while lowest $(2.22 \%)$ was found in the offspring (D717A $\times$ Y3560). The induction of fertile plants in pol CMS maternal parent may be attributed to the hybridization compatibility of $\mathrm{Y} 3380$ and $\mathrm{Y} 3560$ with pol cytoplasmic restoration genes. According to the probability of fertile plants, the induction rate of $Y 3380$ and $Y 3560$ was estimated to vary from $70 \%$ to $100 \%$.

\section{Screening results of SSR molecular markers and SNP chips for induction line F1}

600 pairs of SSR primers were used to differentiate the polymorphism between parental and maternal parents and their progeny (Fig 3, Additional file 4, Additional file 5). When Y3560 was used the parental parent, three pairs of specific primers were amplified with 0068A; four pairs of specific primers were amplified with 0933A and L0933A. Those specific primers were also amplified in their offsprings. Amplification of SSR analysis revealed that induced F1 generation plants were consistent with maternal band type, without paternal or heterozygous band type. At the same time, combined with the field fertility survey, the M1-1, M1-8 and M1-9, the progeny of $0068 \mathrm{~A} \times \mathrm{Y} 3560$ were tentatively judged as the induced plants. While that of Y3380, three pairs of specific primers were amplified with L0068A, five pairs of specific primers were amplified with 0933A and L0933A, and amplification results showed that in addition to L0068A× Y3380 offspring M4-5, M4-9 revealed heterozygous band type, while remaining offspring and maternal band type. ZS11 was used a parental parent, and 2-3 pairs of specific primers were amplified in each of maternal parent. The amplification of primers revealed that the progeny contained the band type of both parents, hence all were hybrid plants. Since the polymorphic primers were not enough, so 6K SNP chip analysis covering more marker sites in whole of genome should be used to verify whether the progeny of each combination is homozygous or heterozygous induced plant? In view of the fact that ploidy, morphology and SSR molecular marker identification would not produce accurate results, 62 plants were selected for $6 \mathrm{~K}$ SNP chip analysis (Additional files 6 ). The results of SNP homozygosity and genetic similarity are given in Additional file 7 and the 7-materials (Additional files 8) used this study showed a homozygosity rate that ranges from $97.93 \% \sim 99.29 \%$. Such higher homozygosity rate was close to ZS11 as control (99.16\%), suggesting that these seven maternal parents were homozygous. The homozygosity rate of F1 hybrid was 63.73\% 68.74\%, and genotyping (Fig 4d, Additional file 9c, Additional file 10e) also indicated that these offspring were F1 hybrids produced by ZS11 as the paternal parent. At the same time, the homozygosity rate of some F1 plants after induction ranged from 56.39\% 78.87\%, and genotyping (Fig 4a-c, Additional file 9a-b, Additional file 10a-d) was used to identify the F1 as hybrid or partial chromosome hybrid offspring. The rate of homozygous SNP sites in the remaining single plants ranged from 98.48\% 99.33\%. Genotyping (Fig 4a-c, Additional file 9a-b, Additional file 10a-d) confirmed that these F1 were homozygous plants. Therefore, the analysis of the homozygous rate between different individual plants showed that used the $\mathrm{DH}$ lines as the paternal parent, the offspring produced by the homozygous rate of about $60 \%$ were hybrid offspring, and the homozygous rate greater than $95 \%$ was the 
induced offspring. Subsequently, we analyzed the genetic distance between the maternal parent and 62 materials, and calculated the genetic similarity rate between the maternal parents and their $\mathrm{F} 1$ offspring. The genetic similarity rate between hybrid offspring and the maternal parent was $64.64 \% \sim 68.74 \%$, while that of genetic similarity rate between the induced homozygous individual plant and the maternal parent was as high as $99.33 \%$.

\section{Genotyping revealed paternal chromosome hybridization and infiltration during induction process}

In order to better understand the paternal chromosome hybridization and infiltration in induction process, homozygous SNP sites with parental differences were screened and genotyping revealed SNP sites in the offspring (Fig 4, Additional file 9, Additional file 10, Additional file 11). The number of homozygous SNP sites in parental differences ranged from 873-1227 (Table 1, Fig 5a, Additional file 11). Taking the maternal parent 0933 as an example, compared with the hybrid combination 0933A× ZS11 (Fig 4d), 97.91\% heterozygosity, when 0933B (Fig 4a) and 0933A (Fig 4b) were the maternal parent, genotype of the induced offspring was consistent with those of the maternal parent. Hybridization of $33.84 \% ~ 44.26 \%$ paternal genes and paternal infiltration rate of $0.09 \sim 0.19 \%$ were observed in the hybrid offspring. When L0933A (Fig 4c) was used as maternal parent, the hybrid offspring revealed $37.87 \% \sim 53.03 \%$ (Table 1) paternal genes heterozygosity and $0.09 \% \sim 0.18 \%$ (Table 1) paternal introgression. These results indicated that the induction efficiency was influenced by cytoplasm genotype, and the hybridization of ogu cytoplasmic genotype was more prone to occur in the maternal parent when the karyogene was same. When the cytoplasmic genotype was pol ( $0068 \mathrm{~A}$, 0933A, D717A), the offspring of 0068A (33.82\% 47.10\%) (Table 1) were more likely to cross with the paternal parent; and the cytoplasmic type was nap (0933B, D717B), the offspring of D717B $(24.84 \%$ $42.86 \%$ ) (Table 1) were most likely to infiltrate the paternal gene; while the cytoplasm genotype was ogu (L0068A, L0933A), the L0933A was easier to cross or exchange with the paternal parent, and was more likely to be on the $\mathrm{C}$-genome (Fig $5 \mathrm{~b}$ ), indicating that the cytoplasm genotype was the same, the induction effect was affected by the maternal parent nuclear genotype. In conclusion, the induction efficiency is influenced by both the maternal karyogene and the cytoplasm genotype, and karyogene > cytoplasmic genotype.

Table 1 Statistics of genotyping SNP sites. 
source sample SNP Heterozygous Heterozygositymaternal maternal paternal number paternal name number number rate $(\%)$ number infiltration rate $(\%)$ rate $(\%)$

\begin{tabular}{|c|c|c|c|c|c|c|c|c|}
\hline \multirow[t]{6}{*}{$\overline{Y 3560}$} & M1-1 & 1174 & 551 & 46.93 & 622 & 52.98 & 1 & 0.09 \\
\hline & M1-2 & 1174 & 553 & 47.10 & 619 & 52.73 & 2 & 0.17 \\
\hline & M1-3 & 1174 & 539 & 45.91 & 634 & 54.00 & 1 & 0.09 \\
\hline & M1-8 & 1174 & 0 & 0.00 & 1174 & 100.00 & 0 & 0.00 \\
\hline & M1-9 & 1174 & 2 & 0.17 & 1172 & 99.83 & 0 & 0.00 \\
\hline & M1-10 & 1174 & 397 & 33.82 & 777 & 66.18 & 0 & 0.00 \\
\hline \multirow[t]{4}{*}{$\times Y 3380$} & M4-3 & 1088 & 324 & 29.78 & 762 & 70.04 & 2 & 0.18 \\
\hline & M4-4 & 1088 & 0 & 0.00 & 1088 & 100.00 & 0 & 0.00 \\
\hline & M4-5 & 1088 & 626 & 57.54 & 460 & 42.28 & 2 & 0.18 \\
\hline & M4-9 & 1088 & 702 & 64.52 & 385 & 35.39 & 1 & 0.09 \\
\hline ZS11 & M5-1 & 1115 & 1106 & 99.19 & 3 & 0.27 & 6 & 0.54 \\
\hline \multirow[t]{6}{*}{ Y3560 } & M31-1 & 1071 & 1 & 0.09 & 1070 & 99.91 & 0 & 0.00 \\
\hline & M31-2 & 1071 & 3 & 0.28 & 1068 & 99.72 & 0 & 0.00 \\
\hline & M31-5 & 1071 & 416 & 38.84 & 654 & 61.06 & 1 & 0.09 \\
\hline & M31-7 & 1071 & 1 & 0.09 & 1070 & 99.91 & 0 & 0.00 \\
\hline & M31-8 & 1071 & 474 & 44.26 & 595 & 55.56 & 2 & 0.19 \\
\hline & $\begin{array}{l}\text { M31- } \\
12\end{array}$ & 1071 & 0 & 0.00 & 1071 & 100.00 & 0 & 0.00 \\
\hline \multirow[t]{6}{*}{ Y3380 } & M30-1 & 1038 & 2 & 0.19 & 1036 & 99.81 & 0 & 0.00 \\
\hline & M30-2 & 1038 & 438 & 42.20 & 599 & 57.71 & 1 & 0.10 \\
\hline & M30-3 & 1038 & 2 & 0.19 & 1036 & 99.81 & 0 & 0.00 \\
\hline & M30-6 & 1038 & 0 & 0.00 & 1038 & 100.00 & 0 & 0.00 \\
\hline & $\begin{array}{c}\text { M30- } \\
10\end{array}$ & 1038 & 1 & 0.10 & 1037 & 99.90 & 0 & 0.00 \\
\hline & $\begin{array}{l}\text { M30- } \\
11\end{array}$ & 1038 & 0 & 0.00 & 1038 & 100.00 & 0 & 0.00 \\
\hline \multirow[t]{4}{*}{ Y3380 } & M28-2 & 1062 & 0 & 0.00 & 1062 & 100.00 & 0 & 0.00 \\
\hline & M28-6 & 1062 & 1 & 0.09 & 1061 & 99.91 & 0 & 0.00 \\
\hline & M28-7 & 1062 & 1 & 0.09 & 1061 & 99.91 & 0 & 0.00 \\
\hline & M28-9 & 1062 & 1 & 0.09 & 1061 & 99.91 & 0 & 0.00 \\
\hline \multirow[t]{5}{*}{$\times \mathrm{Y} 3560$} & M35-1 & 1088 & 18 & 1.65 & 1070 & 98.35 & 0 & 0.00 \\
\hline & M35-7 & 1088 & 17 & 1.56 & 1071 & 98.44 & 0 & 0.00 \\
\hline & M35-9 & 1088 & 577 & 53.03 & 475 & 43.66 & 36 & 3.31 \\
\hline & $\begin{array}{c}\text { M35- } \\
10\end{array}$ & 1088 & 33 & 3.03 & 1055 & 96.97 & 0 & 0.00 \\
\hline & M35- & 1088 & 17 & 1.56 & 1071 & 98.44 & 0 & 0.00 \\
\hline
\end{tabular}




\begin{tabular}{|c|c|c|c|c|c|c|c|c|}
\hline & $\begin{array}{c}\text { M35- } \\
14\end{array}$ & 1088 & 102 & 9.38 & 984 & 90.44 & 2 & 0.18 \\
\hline \multirow[t]{6}{*}{ ×Y3380 } & M34-1 & 1062 & 531 & 50.00 & 529 & 49.81 & 2 & 0.19 \\
\hline & M34-2 & 1062 & 470 & 44.26 & 513 & 48.31 & 79 & 7.44 \\
\hline & M34-3 & 1062 & 509 & 47.93 & 500 & 47.08 & 53 & 4.99 \\
\hline & M34-5 & 1062 & 539 & 50.75 & 522 & 49.15 & 1 & 0.09 \\
\hline & M34-6 & 1062 & 402 & 37.85 & 659 & 62.05 & 1 & 0.09 \\
\hline & M34-9 & 1062 & 79 & 7.44 & 982 & 92.47 & 1 & 0.09 \\
\hline ZS11 & M36-1 & 1053 & 1031 & 97.91 & 2 & 0.19 & 20 & 1.90 \\
\hline xZS11 & M36-1 & 1079 & 1041 & 96.48 & 2 & 0.19 & 36 & 3.34 \\
\hline \multirow[t]{5}{*}{ Y3560 } & M39-2 & 1071 & 465 & 43.42 & 604 & 56.40 & 2 & 0.19 \\
\hline & M39-4 & 1071 & 2 & 0.19 & 1069 & 99.81 & 0 & 0.00 \\
\hline & M39-6 & 1071 & 1 & 0.09 & 1070 & 99.91 & 0 & 0.00 \\
\hline & M39-8 & 1071 & 460 & 42.95 & 610 & 56.96 & 1 & 0.09 \\
\hline & $\begin{array}{c}\text { M39- } \\
13\end{array}$ & 1071 & 480 & 44.82 & 588 & 54.90 & 3 & 0.28 \\
\hline \multirow[t]{4}{*}{ Y3380 } & M38-1 & 873 & 403 & 46.16 & 470 & 53.84 & 0 & 0.00 \\
\hline & M38-2 & 873 & 396 & 45.36 & 477 & 54.64 & 0 & 0.00 \\
\hline & M38-5 & 873 & 388 & 44.44 & 485 & 55.56 & 0 & 0.00 \\
\hline & M38-9 & 873 & 390 & 44.67 & 478 & 54.75 & 5 & 0.57 \\
\hline \multirow[t]{2}{*}{$\bar{Y} 3560$} & M10-1 & 1071 & 408 & 38.10 & 397 & 37.07 & 266 & 24.84 \\
\hline & M10-2 & 1071 & 7 & 0.65 & 605 & 56.49 & 459 & 42.86 \\
\hline \multirow[t]{3}{*}{ Y3380 } & M9-1 & 873 & 43 & 4.93 & 830 & 95.07 & 0 & 0.00 \\
\hline & M9-2 & 873 & 1 & 0.11 & 872 & 99.89 & 0 & 0.00 \\
\hline & M9-3 & 873 & 2 & 0.23 & 871 & 99.77 & 0 & 0.00 \\
\hline ZS11 & M42-1 & 1227 & 1208 & 98.45 & 3 & 0.24 & 16 & 1.30 \\
\hline
\end{tabular}

Bold text represents induced offspring. Italic text is the hybrid offspring of ZS11 as the paternal parent.

\section{Analysis of the interaction effect between the inducer line and the maternal karyogene}

Since maternal karyogene and cytoplasm genotype jointly affects the induction efficiency, it is not clear whether the inducer hybridization and infiltration of the sites is random. Based on the comparison of SNP in genotyping (Table 1, Fig 5a), it was found that the SNP sites of normal hybrid offspring with ZS11 as the paternal parents were heterozygous with the exception of individual sites infiltrated by the paternal parents, and the heterozygous rate were $96.48 \%$ 99.19\% (Table 1), and the paternal infiltration rate was $0.54 \%$ $3.34 \%$ (Table 1). While using the inducer as the paternal parent, although hybrid progeny will be produced, the 
heterozygous rate of these hybrid progeny is only $29.78 \% \sim 53.03 \%$ (Table 1), and most of the sites are the same as the maternal parent. Subsequent analysis of the chromosomes in which these hybridization sites are located found that when ZS11 is the paternal parent, hybridization mainly occurs on the $\mathrm{C03}$ and $\mathrm{C0} 4$ chromosomes of the $\mathrm{C}$ genome, and the heterozygosity rate is as high as $99.2 \% \sim 100 \%$ (Table 1); When the inducer was used as the paternal parent, although the same as the normal hybridization, it mainly occurred on the $\mathrm{CO} 3$ and $\mathrm{C0} 4$ chromosomes of the $\mathrm{C}$ genome, but the heterozygosity rate on $\mathrm{C0} 3$ and $\mathrm{C0} 4$ in the hybrid progeny was $28.1 \% \sim 68.14 \%$ and $30.71 \% \sim 86.2 \%$ (Table 1), which were significantly lower than the normal hybridization level. Therefore, the cross produced by using the inducer as the paternal parent is not an ordinary cross.

At the same time, the chromosome where the paternal infiltration site is located is analyzed (Table 1, Fig 5b-c). 0068 and 0933 have similar genetic background (from part of one same parent), when used as maternal parent, the inducer was the paternal parent, the infiltration of the paternal parent gene was more likely to be on the $\mathrm{C} 04$ and $\mathrm{CO} 6$ chromosomes of the $\mathrm{C}$ genome; while ZS11 was the paternal parent, the sites of paternal parent infiltration were mainly on the $\mathrm{C} 05$ and $\mathrm{C0} 6$ chromosomes of the C-genome, and the paternal parent of the inducer is more likely to cause the infiltration (Fig 5b). However, since the site on chromosome $\mathrm{C} 06$ mostly the same site, thus eliminating $\mathrm{C06}$ penetration site on the chromosome, and presumed DH lines is more likely to cause parent gene penetration on the $\mathrm{CO} 4$ chromosome of the $\mathrm{C}$ genome, and the paternal infiltration caused by normal hybridization is more likely to occur on the $\mathrm{C} 05$ chromosome. When the maternal parent was $\mathrm{D} 717$, the paternal parent infiltration sites of the induced was more likely to appear on the C07

chromosome of the $\mathrm{C}$ genome, while the parent of ZS11 was mainly on the A06 chromosome of the A genome and the $\mathrm{C} 06$ chromosome of the $\mathrm{C}$ genome. In summary, the analysis of the paternal cross and infiltration sites, it is indicated that the $\mathrm{DH}$ inducer has an interaction effect with the maternal karyogene, and has the biases on different genomes.

\section{Discussion}

\section{Induction effect of rape DH inducer lines}

When the Maize haploid inducer line induces the maternal plant to produce the haploid, it has obvious maternal genotype influence, the induction efficiency ranges from $2 \%$ to $15 \%$, there is also about $2 \%$ of the paternal gene infiltration phenomenon, and whether the Bassica napus DH inducer lines has a similar situation has not been reported. In this study, two DH inducer lines of Brassica napus, Y3380 and Y3560, were used for pollination of maternal parents with different cytoplasmic genotype, and multiple methods were used for identification (Additional file 12). Identification methods were consistent to reflect the effects of induction and hybridization, fertile offspring, heterozygous offspring marker with SSR, and heterozygous offspring detected with SNP, indicating that the detection results were completely reliable. Combining these test results, it is found that the induction system has different induction effects on different genotype of Brassica napus. When the karyogene were the same, the induction difference was mainly affected by cytoplasmic genotype. In the same cytoplasmic genotype, the induced differences are mainly affected by the karyogene. Therefore, the induction process of DH inducer line has interaction effects with maternal karyogene and cytoplasmic genotype. At same time, it was found that there was a very significant positive correlation between the genetic similarity rate and homozygous rate of SNP sites of induced F1 generation and the maternal parent 
(Additional file 7), Such as F1 of $0068 \mathrm{~A} \times \mathrm{Y} 3560$ and the maternal parent genetic similarity of $99.79 \%$, but its SNP sites homozygous rate and parent SNP sites homozygous rate of $98.79 \%, 97.93 \%$, respectively (Additional file 7); F1 of $0933 A \times$ Y 3560 has a genetic similarity of $98.86 \%$ with the maternal parent, but its SNP sites homozygous rate was $99.23 \%$, while the maternal parent SNP sites homozygous rate was $98.27 \%$ (Additional file 7). It shows that the DH inducer lines can make homozygous parents infinitely closer to homozygous. In addition, there was some heterozygosity in the paternal parent genes of the F1 generation, but the heterozygosity varied greatly, ranging from 0.09 to $64.52 \%$ (Table 1). For the induced offspring, some individual plants will also have a small amount of paternal gene infiltration $(0.09-0.18 \%)$. In the induced offspring, all the po/CMS and nap cytoplasm did not have paternal gene infiltration, while in the induced offspring of ogu CMS as the maternal parent, there was low frequency infiltration of the DH inducer gene, and high frequency hybridization. (Fig 5a, Table 1). These results indicated that the DH inducer line gene was more likely to penetrate into the progeny when the mother containing ogu cytoplasm was induced.

\section{Mechanism analysis of induction of DH inducer line}

The study of inducing vivo induced plants to produce haploid or double haploid has been explored in crops such as maize [20-22], Brassica napus [17, 18]. However, the mechanism of the induction function of the DH inducer lines is not yet clear, and it is generally believed that uniparental chromosome elimination $[14,23$, 24] found in maize that inducer genes were infiltrated into haploids $X u$ et al [25] confirmed that double fertilization occurred during the induction of maize haploids, and showed that chromosome elimination was the basis of maize haploid induction; Zhao et al [22] Found that within 7 days after pollination, most of the chromosomes of the inducible lines were excreted from the cell, and about $44 \mathrm{Mb}$ of paternal parent chromosomal fragments were also found in the haploid offspring, further confirming the phenomenon of chromosome infiltration. Therefore, based on the investigation results of the mechanism of maize haploid induction line, this is similar to our findings in Brassica napus of DH inducer.

In this study, allo-octoploid Y3380 and Y3560 were used for pollination of tetraploid Brassica napus, and the progenies were tetraploid. Except for fertility, the progenies were almost the same as the maternal parents, but were significantly different from the paternal parents. From the results of genotyping (Table 1), the SNP hybridization rate of ZS11 as a paternal parent hybrid is over $96.48 \%$, and the maternal parent infiltration rate is $0.19 \% \sim 0.27 \%$. And the use of inducer as paternal parent, SNP hybrid highest rate of $64.52 \%$, the lowest was $0 \%$, and the maternal parent infiltration rate is $35.39 \%$ 100\%, indicating that even if there is hybridization between the inducer and maternal parent, it is not general cross, but partial chromosome or genes cross between the inducer and maternal parent. Therefore, we speculate that the reason for the induction of double haploid in Brassica napus may also be the selective elimination of inducer chromosomes. A little part of Chromosome or large fragment cross with the maternal parent may be caused by incomplete or

partial chromosome loss of inducer. The infiltration of a small number of inducer genes in the progeny may be caused by gene exchange. Further studies are needed to determine whether the induction mechanism of Y3560 and Y3380 is related to some specific genes.

\section{Application of DH inducer in Brassica napus breeding}


Based on the above research results, due to the hybridization or gene infiltration of DH inducer to maternal chromosome (but the genotype is highly consistent with maternal karyogene, the maternal infiltration rate is $90.44 \%$ to $99.91 \%)$, these conditions may slightly change the maternal inheritance characteristics, and the overall consistency, especially for the same karyogene and different cytoplasmic maternal parent, hybridization and gene infiltration are quite different. We boldly predict that the $\mathrm{DH}$ inducer can provide a new model for the innovation of Brassica napus germplasm resources. The innovation the germplasm resources were from $\mathrm{DH}$ inducer specific gene infiltration, and we observed in the field that the purple leaf mutation was found during the induction of ogu cytoplasmic Brassica napus (the parent does not have purple traits) (Fig 2c). It is speculated that the infiltration of the inducer gene fragment or transposon during the induction process leads to the acquisition of certain functions of the maternal parent. Therefore, the phenomenon that the infiltration of the inducer gene enables the plant to obtain certain functions can be applied to development of rape germplasm resources, to find in-depth special sites, and to modify the maternal genes at specific sites. The application of rape $\mathrm{DH}$ inducer can accelerate and change the rape breeding model, and create new ideas for development of germplasm resources, which have huge application potential and practical value.

\section{Conclusions}

This study explored the induction characteristics of two double-haploid inducible lines of Brassica napus. It was found that when the induced lines were used as the paternal parent to pollinate the maternal parent, the hybrid offspring showed heterogeneous hybridization of the paternal parent gene; and some plants of the induced offspring had a small amount paternal gene infiltration. And according to the different types of maternal parent, there are differences in induction. When the karyogene are the same, the induction differences are mainly affected by cytoplasmic genes; when the cytoplasmic genes are the same, the induction differences are mainly affected by karyogene genes. The induction process of the inducible line interacts with the maternal genotype and cytoplasmic type. Use this interaction effect to provide new insights for the innovation of Brassica napus germplasm resources.

\section{Methods}

\section{Planting materials and hybrid combinations}

The DH inducer lines were Y3380 and Y3560 (AAAACCCC, $2 n=8 x \approx 76$ ), new rapeseed varieties artificially bred by of Chengdu Academy of Agriculture and Forestry Sciences in 2011 [18], which were identified as allooctoploid by cytology and flow cytometry, and were used as the paternal parent. The maintainer lines (0933B, D717B, nap cytoplasm genotype) were the maternal parent and were more than 15 generations old normal tetraploid high-generation inbred lines. pol CMS (0068A, 0933A, D717A) and ogu CMS (L0068A, L0933A) (AACC, $2 n=38$ ) backcrossed to maintainer lines for 12-15 generations, therefore have the same karyogene.

For all types of maternal parents (maintainer lines, pol CMS, ogu CMS) used in this experiment, the same number represents the same karyogene, and only cytoplasmic genotype differences (Additional file 8). The collection and breeding of the Brassica napus materials we use has obtained local permits in China, which is in line with national policies. The Brassica napus used in this study is commonly grown oil crop used species native to China and does not fall under the Nagoya protocol. All plant materials including, sterile lines, maintainer lines with induction lines Y3380, Y3560 and ZS11 (control), were grown under the same conditions 
at the experimental field in Wenjiang (E103.83, N30.70), Chengdu, China, in October 2016, pollinated artificially at the flowering stage in March 2017 and harvested by mixed threshing. All experimental combinations had three replications. In October 2018, we raised all received seeds in the nursery pot and transplanted survived plants to the field.

\section{Flow cytometry to identify ploidy}

By selecting young leaves of the plants [26], we randomly choose 15 induction lines F1 of each combination for flow cytometry and tested a minimum of 15 plants for each. We crossed a control (ZS11 as the paternal parent) to the F1 generation (maternal parent) to test three randomly selected strains and perform the statistical analysis.

\section{Induced progeny plant type and fertility identification}

According to the characteristics of sterile lines, fertility as marker character, identification and statistics were carried out by artificial observation in the field. Among them, fertile plants had full anthers and normal pollen, while sterile lines showed different characteristics according to different types. The morphology of pol CMS plants was not significantly different from that of fertile plants, but anthers were atrophied, without pollens or containing few pollens at low temperature [27]. The plant morphology of ogu CMS was not significantly different from fertile rapeseed plants, but the stamens were differentiated with thin anthers and did not produce normal pollen. We identified and counted the fertility and plant types of each F1 strain and photographed selected representative plants.

\section{DNA extraction and PCR amplification}

The total genomic DNA of leaves was extracted using the CTAB method. We grinded fresh leaves quickly in liquid nitrogen, added in $900 \mu \mathrm{l}$ CTAB extract, and then heated in a water bath for 45 min, followed by placing on a shaker (80 rpm) for 15 min for mixing well. Then centrifuged the samples at 12000 rpm for 10 minutes, aspirated the supernatant, added an equal volume of pre-cooled isopropanol and placed on ice for $30 \mathrm{~min}$. All samples were then centrifuged at $12000 \mathrm{rpm}$ for 5 minutes, washed twice with $70 \%$ and absolute ethanol, dissolved in $100 \mathrm{uL} \mathrm{ddH2O}$, and finally, DNA quality was detected by $0.8 \%$ agarose gel electrophoresis. The PCR reaction program is $94^{\circ} \mathrm{C}$ for $4 \mathrm{~min}, 94^{\circ} \mathrm{C}$ for the $30 \mathrm{~s}, \mathrm{TM} 30 \mathrm{~s}, 72^{\circ} \mathrm{C}$ for $45 \mathrm{~s}, 72^{\circ} \mathrm{C}$ for $8 \mathrm{~min}, 35 \mathrm{Cycle}$. And $2 \%$ to $2.5 \%$ agarose gel electrophoresis for detection.

\section{SNP chip identification and analysis}

We used the 6K Infinium SNP chip, which is evenly distributed across the rapeseed genome (Additional file 13, for distribution of the chip on the rape chromosome), to analyze the parental gene loci in the progeny. The reference genome was Brassica napus_v4.1

(http://brassicadb.org/brad/datasets/pub/Genomes/Brassica_napus/). The quality control of SNP sites was performed on samples with DQC $\geq 0.82$ and $C R \geq 95$. Finally, 3816 SNP sites with useful polymorphism were screened out, accounting for $75.44 \%$ of the total labeled sites. These loci were analyzed by using related software, and the homozygosity rate of individual gene sites and the genetic similarity rate of maternal parents were compared. By using Power Maker V3.25 software, we calculated the genetic distance between 
the maternal parents. We obtained the genetic similarity rate for which the formula is $S=\mathrm{Nxy} / \mathrm{Nx}+\mathrm{Ny}(\mathrm{Nxy}$ is the same genotype in the two samples, $\mathrm{Nx}$ and $\mathrm{Ny}$ are the number of genotypes in $\mathrm{X}$ and $\mathrm{Y}$ samples, respectively). SNP gene analysis was based on the SNP locus of each pollination combination that was different and homozygous for the parents. The SNP of the same locus in the offspring using R studio for genotyping (Additional file 11). If the SNP banding pattern in offspring is the same as the paternal parent, it is judged to be infiltrated and consistent with the maternal parent. It is judged to be induced, and the parental heterozygous band type is judged to be hybrid. The 6Kchip detection was completed by China Golden Marker Biology Co., Ltd (Additional file 6).

\section{Declarations}

\section{Acknowledgements}

All authors are grateful to the laboratory members for help, advice and discussion. All authors are grateful to the editors and referees for their valuable comments to improve our manuscript.

\section{Authors' contributions}

SHF, SXG, and WZ, designed the experiments. YTM, ZDZ, LJH, AA, XL, YZ, YL, PZX,JY ZL, HRS, JSW, WZG, ZQ, LRT, ZMK, RT, ZJZ and LZ performed the experiments. WZ wrote the manuscript. SHF edited the manuscript. All authors read and approved its content.

\section{Funding}

This work was supported by the National Key Research and Development Program (2016YFD0101300), the Rapeseed Breeding Program in Sichuan Province during the 13th Five-Year Plan Period, the Sichuan Innovation Team Program, the Major Science and Technology Program in Sichuan Province (2018NZDZX0003), and the Chengdu Applied Technology Research and Development Program.

\section{Availability of data and materials}

The datasets supporting the conclusions of this article are included within the article and its additional files.

\section{Ethics approval and consent to participate}

Not applicable.

\section{Consent for publication}

Not applicable.

\section{Competing interests}

The authors declare that they have no competing interests.

\section{References}


1. Chalhoub B, Denoeud F, Liu S, Parkin I, Tang H, Wang, X, Chiquet J, Belcram H, Tong C, Samans B, Corréa $\mathrm{M}$ et al: Early allopolyploid evolution in the post-Neolithic Brassica napus oilseed genome Science 2014, 345(6202):1255-1255.

2. Hollister JD: Polyploidy: adaptation to the genomic environment. New Phytologist 2015, 205(3):10341039.

3. Charne DG, Pukacki P, Kott LS, Beversdorf WD: Embryogenesis following cryopreservation in isolated microspores of rapeseed (Brassica napus L.). Plant Cell Reports 1988, 7(6):407-409.

4. Fan Z, Armstrong KC, Keller WA: Development of microspores in vivo and in vitro in Brassica napus $L$. Protoplasma 1988, 147(2-3):191-199.

5. Lichter R: Induction of Haploid Plants From Isolated Pollen of Brassica napus. Zeitschrift für Pflanzenphysiologie 1982, 105:427-434.

6. Gu HH, Hagberg P, Zhou WJ: Cold pretreatment enhances microspore embryogenesis in oilseed rape (Brassica napus L.). Plant Growth Regulation 2004, 42(2):137-143.

7. Lionneton E, Beuret W, Delaitre C, OchattM S, Rancillac: Improved microspore culture and doubled-haploid plant regeneration in the brown condiment mustard (Brassica juncea). Plant Cell Reports 2001, 20(2):126-130.

8. Mllers C, Iqbal MCM, Rbbelen G: Efficient production of doubled haploid Brassica napus plants by colchicine treatment of microspores. Euphytica 1994, 75(1):95-104.

9. Lashermes P, Beckert M: Genetic control of maternal haploidy in maize (Zea mays L.) and selection of haploid inducing lines. Theoretical \& Applied Genetics 1988, 76(3):405-410.

10. Zhong, C X: Centromeric Retroelements and Satellites Interact with Maize Kinetochore Protein CENH3. Plant Cell 2002, 14(11):2825-2836.

11. Sanei M, Pickering R, Kumke K, Nasuda S, Houben A: Loss of centromeric histone H3 (CENH3) from centromeres precedes uniparental chromosome elimination in interspecific barley hybrids. Proceedings of the National Academy of Sciences 2011, 108(33):13373-13374.

12. Zhang T, Talbert PB, Zhang W, Wu Y, Yang Z, Henikoff JG, Henikoff S, Jiang J: From the Cover: PNAS Plus: The CentO satellite confers translational and rotational phasing on cen $\mathrm{H} 3$ nucleosomes in rice centromeres. Proceedings of the National Academy of Sciences of the United States of America 2013, 110(50):E4875-E4883.

13. Chenxu L, Xiang L, Dexuan M, Yu Z, Chen C, Xin D, Xiaowei X, Baojian C, Wei L, Liang L et al: A 4-bp Insertion at ZmPLA1 Encoding a Putative Phospholipase A Generates Haploid Induction in Maize. Molecular plant 2017, 10(3):520-522.

14. Ravi, Chan, Swl: Haploid plants produced by centromere-mediated genome elimination. Nature 2010, 464(7288):615-U180.

15. Karimi-Ashtiyani R, Ishii T, Niessen M, Stein N, Andreas H: Point mutation impairs centromeric CENH3 loading and induces haploid plants. Proceedings of the National Academy of Sciences of the United States of America 2015, 112(36):11211-11216.

16. Britt AB, Kuppu S: Cenh3: An Emerging Player in Haploid Induction Technology. 2016, 7(357). 
17. Liqin Y, Zhendong Z, Xuan L, Liangjun H, Yu L, Annaliese S. Mason, Jin Y, Xianhong G, Yan L, Jisheng W et al: Genome-Wide Duplication of Allotetraploid Brassica napus Produces Novel Characteristics and Extensive Ploidy Variation in Self-Pollinated Progeny. G3 2020, 10:3687-3699.

18. Fu S, Yin L, Xu M, Li Y, Wang M, Yang J, Fu T, Wang J, Shen J, Ali A et al: Maternal doubled haploid production in interploidy hybridization between Brassica napus and Brassica allooctaploids. Planta 2018, 247(1):113-125.

19. Ranney T: Polyploidy From Evolution to New Plant Development. researchgate 2006, 56:137-142.

20. Li L, Xu X, Jin W, Chen S: Morphological and molecular evidences for DNA introgression in haploid induction via a high oil inducer CAUHOI in maize. Planta 2009, 230(2):367-376.

21. Qiu F, Liang Y, Li Y, Liu Y, Wang L, Zheng Y: Morphological, cellular and molecular evidences of chromosome random elimination in vivo upon haploid induction in maize. Current Plant Biology 2014, $1(\mathrm{C}): 83-90$.

22. Xin Z, Xiaowei X, Hongxia X, Shaojiang C, Jin. W: Fertilization and Uniparental Chromosome Elimination during Crosses with Maize Haploid Inducers. Plant Physiology 2013, 163:721-731.

23. Ishii T, Karimi-Ashtiyani R, Houben A: Haploidization via Chromosome Elimination: Means and Mechanisms. Annual review of plant biology 1981, 67(1):421.

24. Kim S, Armstrong K, Fedak G, Ho K, Park NI: A microsatellite sequence from the rice blast fungus (Magnaporthe grisea) distinguishes between the centromeres of Hordeum vulgare and $\mathrm{H}$. bulbosum in hybrid plants. Genome 2002, 45(1):165-174.

25. Xu. X, Li. L, Dong. X, Jin. W, E. MA, Chen S: Gametophytic and zygotic selection leads to segregation distortion through in vivo induction of a maternal haploid in maize. Journal of Experimental Botany 2013, 64(4):1083-1096.

26. Takahira J, Cousin A, Nelson MN, Cowling WA: Improvement in efficiency of microspore culture to produce doubled haploid canola (Brassica napus L.) by flow cytometry. Plant Cell Tissue \& Organ Culture 2011, 104(1):51-59.

27. Guangsheng Y, Tingdong F: The Inheritance of Polima Cytoplasmic Male Sterility in Brassica napus L. Plant Breeding 2010, 104(2):121-124.

\section{Additional Information}

\section{Additional Files}

Additional file 1. Flow cytometry diagram of induced line Y3560, maternal parent 0068A and induced offspring.

Additional file 2. Flow cytometry results statistics.

The ploidy of rape was calculated by fluorescence intensity, and the peak value range represents the fluorescence signal value of mitotic G1 phase of flow cytometry. There are some differences in the fluorescence signal value of each material under the same ploidy, and there significant differences in the fluorescence signal value between ploidy. 
Additional file 3. Fertility identification results.

Additional file 4. Amplification results of SSR primers of some parents and F1 individual plants.

a: Amplification of SSR specific primer $\mathrm{H} 57$ in the paternal inducible line Y3560 (as paternal parent), 0068A (as maternal parent) and F1 progeny, 0068A×Y3560 progeny, from left to right are M1-1, M1-2, M1-3, M1-8, M1-9, M1-10; amplification of specific SSR primer H172 in parents and hybrid progeny (0068A×ZS11). b: Amplification of SSR specific primer H57 in the paternal inducible line Y3380 (as paternal parent), L0068A (as maternal parent) and F1 progeny, L0068A×Y3380 progeny, from left to right are M4-3, M4-4, M4-5, M4-9; amplification of specific SSR primer H172 in parents and hybrid progeny (L0068A×ZS11). C: Amplification of SSR specific primer $\mathrm{H} 57$ in the paternal inducible line (as paternal parent), D717A (as maternal parent) and F1 progeny, D717A×Y3560 progeny, from left to right are M39-2, M39-4, M39-6, M39-8, M39-13; D717A×Y3380 progeny, from left to right are M38-1口M38-2ロM38-5, M38-9. d: Amplification of specific SSR primer H38 in parents and hybrid progeny (D717AxZS11). The samples derive from the same experiment and the full-length original gel is included in Additional file 5 .

Additional file 5. Original image of agarose gel electrophoresis detection.

a: Original agarose gel electrophoresis detection image of Fig3a-b. b: Original agarose gel electrophoresis detection image of Fig3c-d, except that the first band is a duplicate of the maternal parent L0068A, the others are the same as in Fig 3c-d. c-d: Original agarose gel electrophoresis detection image of Additional file 4.

Additional file 6. 6K SNP chip raw data.

Additional file 7. SNP homozygosity rate and inheritance rate.

Additional file 8. Test material information.

The same number of materials used represents the same karyogene, " $\mathrm{A}$ " represents sterile line, " $\mathrm{B}$ " represents maintainer line, pol CMS was bred by backcross with 15 generations of maintainer line, and ogu CMS was bred by backcross with 12 generations of the same maintainer line, only cytoplasmic genotype differences, but 0068 and 0933 have similar sources. The collection and breeding of the Brassica napus materials we use has obtained local permits in China, which is in line with national policies. The Brassica napus used in this study is commonly grown oil crop used species native to China and does not fall under the Nagoya protocol.

Additional file 9. Genotyping diagram of induced line before and after induction of 0068 .

a-c: respectively, are the genotyping diagrams of parent and progeny before and after induction of $0068 \mathrm{~A} \times \mathrm{Y} 3560, \mathrm{~L} 0068 \mathrm{~A} \times \mathrm{Y} 3380,0068 \mathrm{~A} \times \mathrm{ZS} 11$, and the numbered band $\mathrm{M}$ is the progeny plant.

Additional file 10. Genotyping diagram of induced line before and after induction of D717.

a-e: for the genotyping diagrams of parents and progeny before and after induction of D717A $\times$ Y 3560 , $D 717 A \times Y 3380, D 717 B \times Y 3560, D 717 A \times Y 3380$, and D717A $\times Z S 11$, respectively. The band $M$ in the number is the progeny plant. 
Additional file 11. Genotyping raw data.

Additional file 12. Statistics of identification results of different identification methods.

Additional file 13. SNP site distribution map.

The blue dots represent the distribution of SNP sites on chromosomes A01-A10 and C01-C09 in Brassica napus. The $6 \mathrm{k}$ (total 5127 sites) SNP chip is well distributed on 19 chromosomes.

\section{Figures}
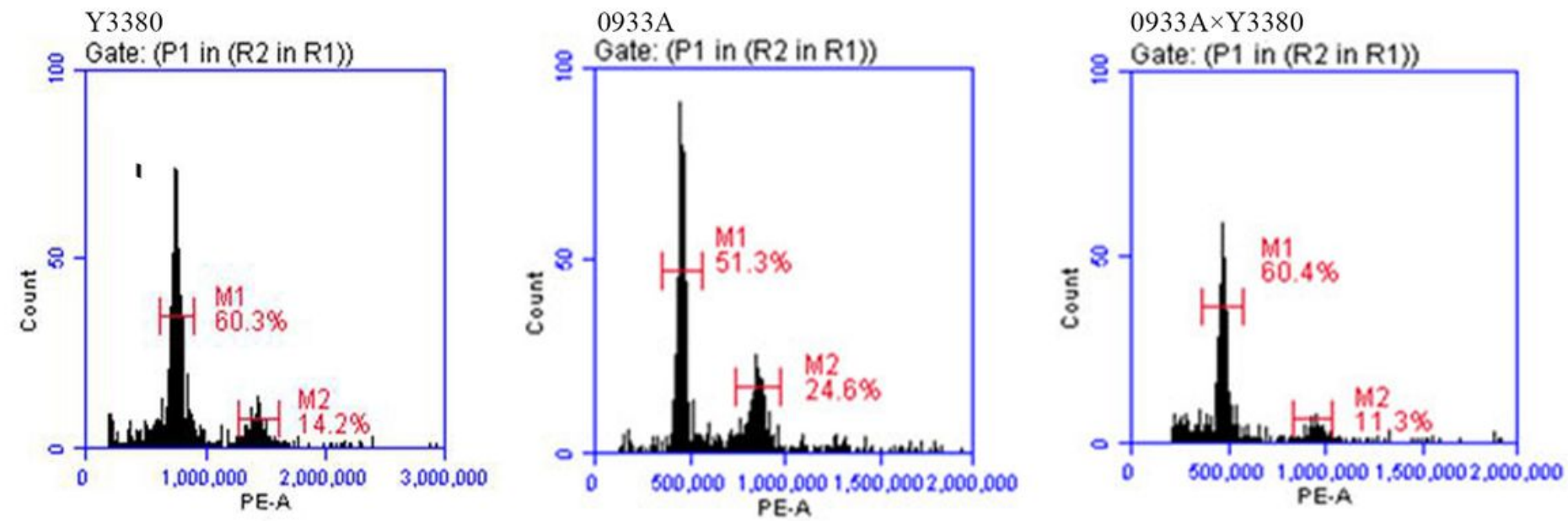

\section{Figure 1}

Flow cytometry diagram of induced line $\mathrm{Y} 3380$, maternal parent 0933A and their induced offspring (0933A×Y3380). 


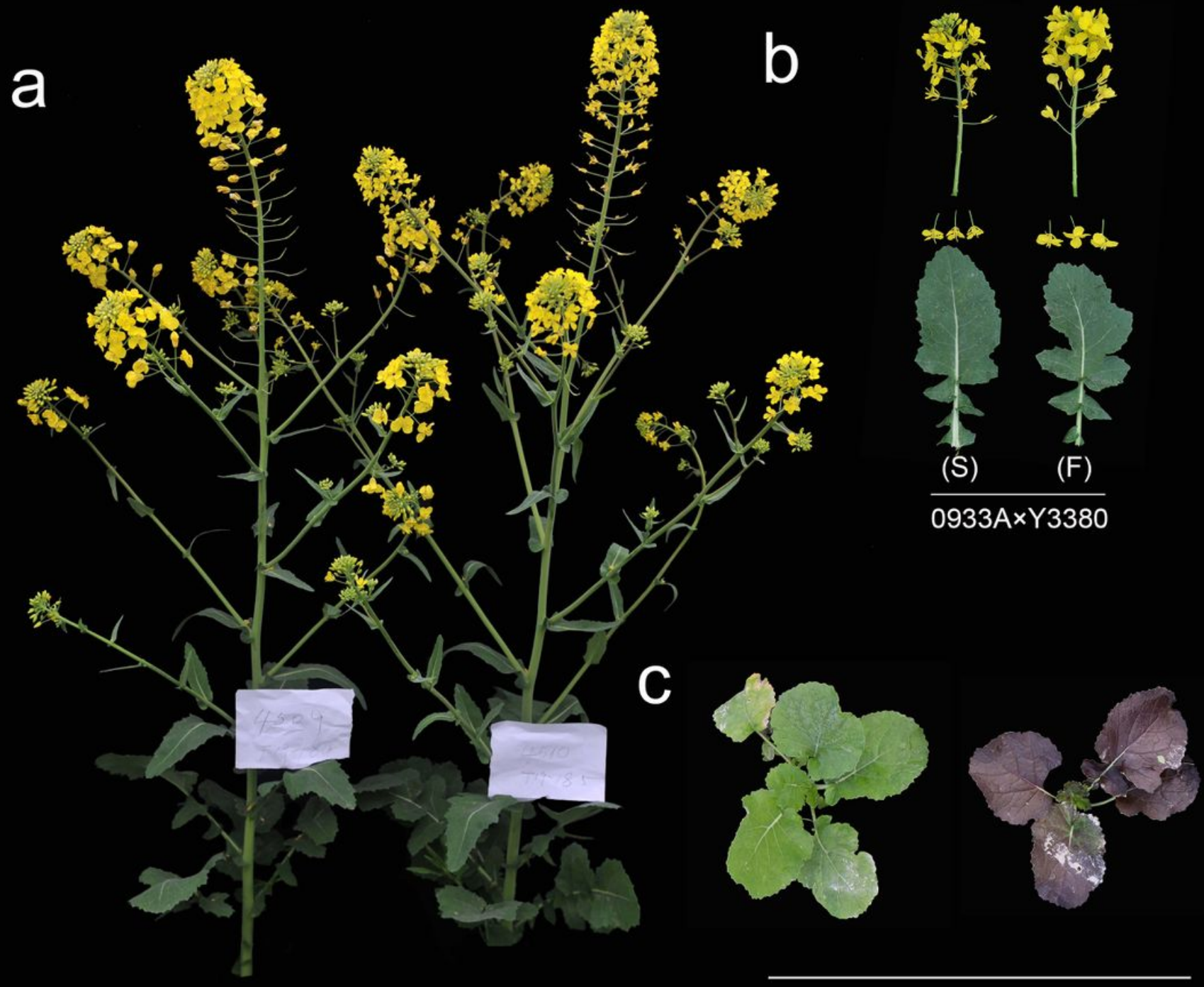

0933B $\times Y 3380(F) \quad 0933 A \times Y 3380(S)$

L0933A×Y3380

\section{Figure 2}

Comparison of phenotype in florescence flower and leaf of 0933. a: Phenotype of maternal parent 0933B $\times$ Y3380 and 0933A×Y3380 (F: fertility, S: sterility). b: Phenotype of inflorescence and flower of 0933A×Y3380 (F: fertility, S: sterility). c: Phenotype of leaf of induction line L0933A progeny with leaf contrasts in purple color. 


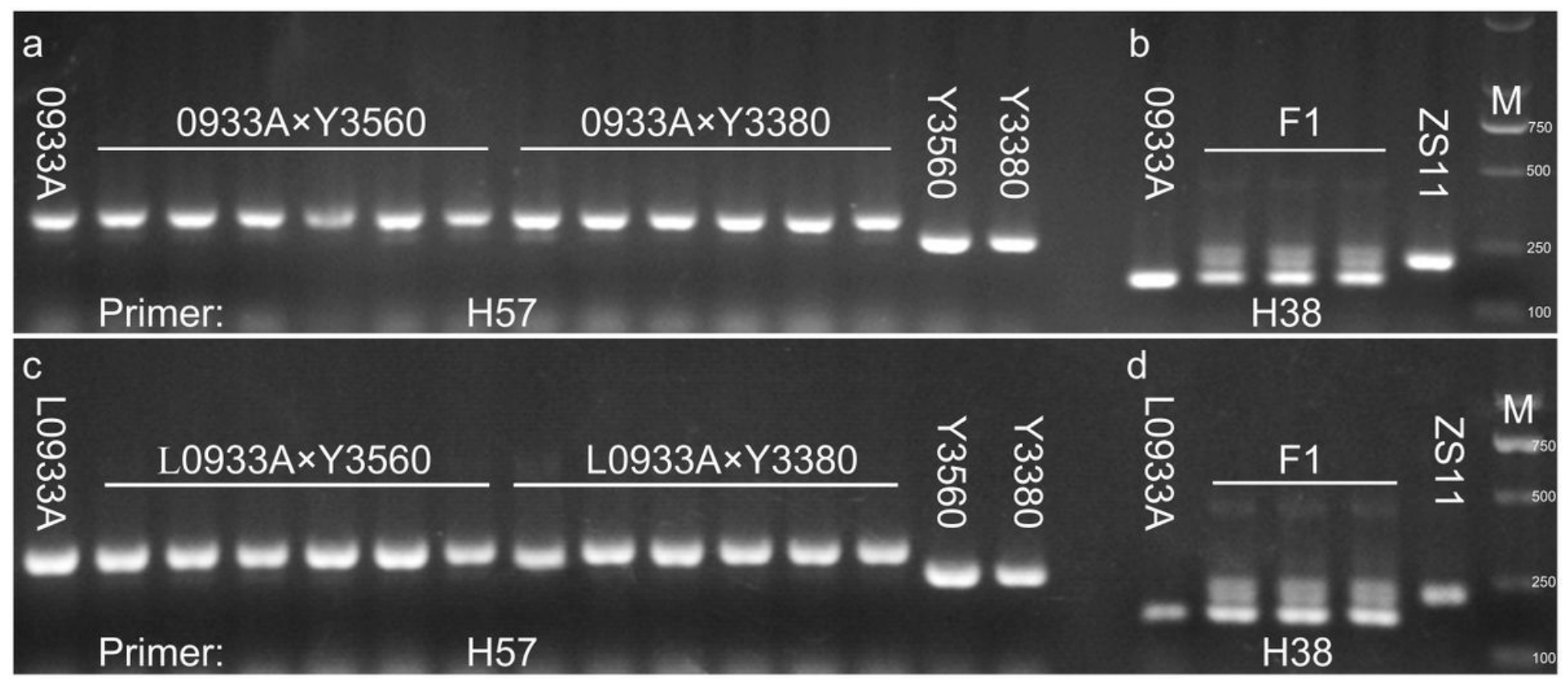

\section{Figure 3}

Amplification results of SSR primers of some parents and F1 individual plants. a: Amplification of SSR specific primer H57 in the paternal inducible line (as paternal parent), 0933A (as maternal parent) and induced progeny, 0933A×Y3560 progeny, from left to right are M31-1, M31-2, M31-5, M31-7, M31-8, M31-12;

0933A×Y3380 progeny, from left to right are M30-1, M30-2, M30-3, M30-6, M30-10, M30-11. b: Amplification of specific SSR primer H38 in parents and hybrid progeny (0933A×ZS11). c: Amplification of SSR specific primer $\mathrm{H} 57$ in the paternal inducible line (as paternal parent), L0933A (as maternal parent) and induced progeny, L0933A×Y3560 progeny ,from left to right are M35-1, M35-7, M35-9, M35-10, M35-12, M35-14; L0933A×Y3380 progeny, from left to right are M34-1, M34-2, M34-3, M34-5, M34-6, M34-9. d: Amplification of specific SSR primer H38 in parents and hybrid progeny (L0933A×ZS11). The samples derive from the same experiment and the full-length original gel is included in Additional file 5. 

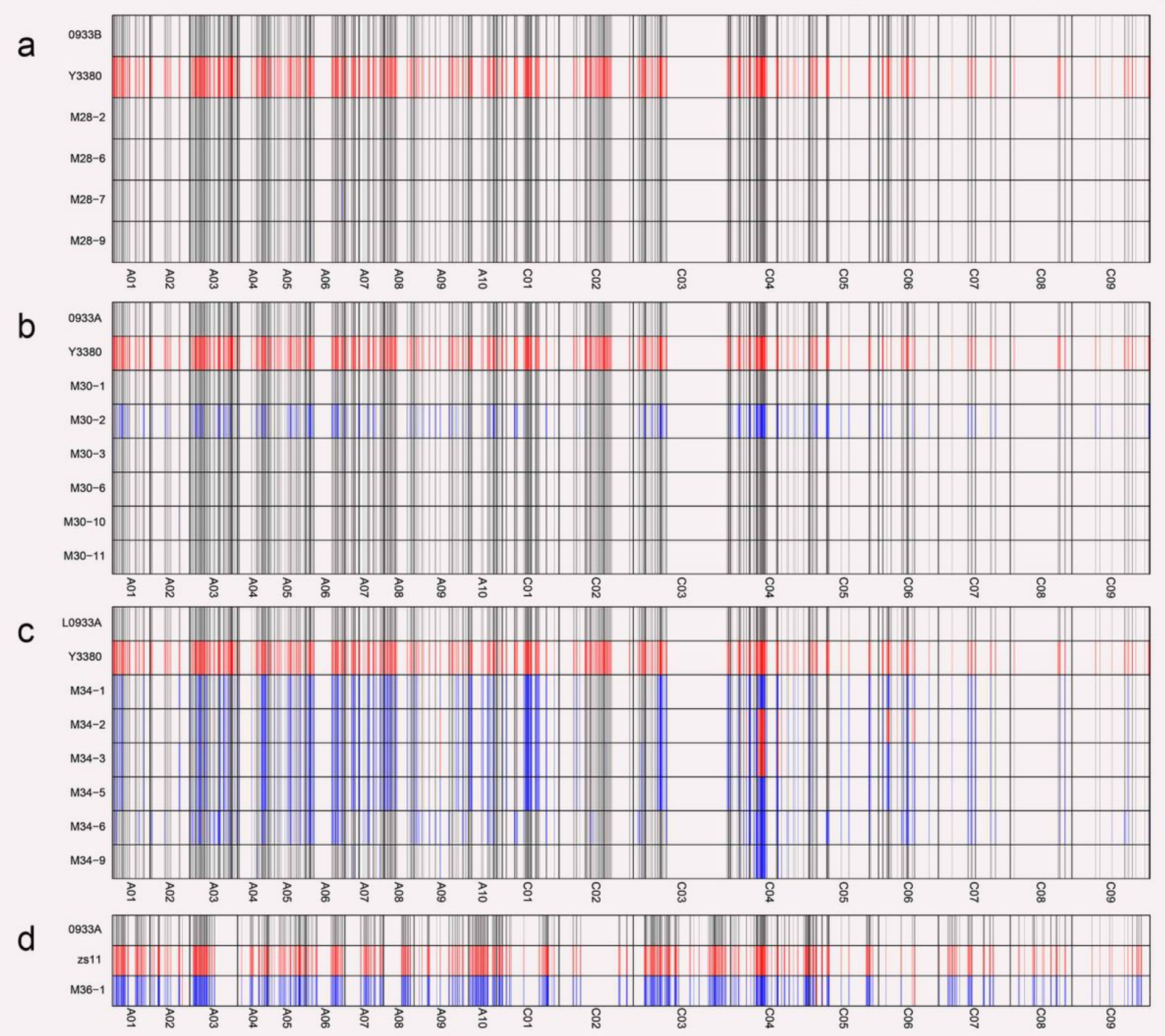

\section{Figure 4}

Genotyping diagram of induced line before and after induction. a: 0933B $\times$ Y3380 . b: 0933A $\times$ Y3380. c: L0933A×Y3380. d: 0933A×ZS11. Schematic diagram of the genotyping of parents and progeny, the numbered band $\mathrm{M}$ is the progeny plant. 

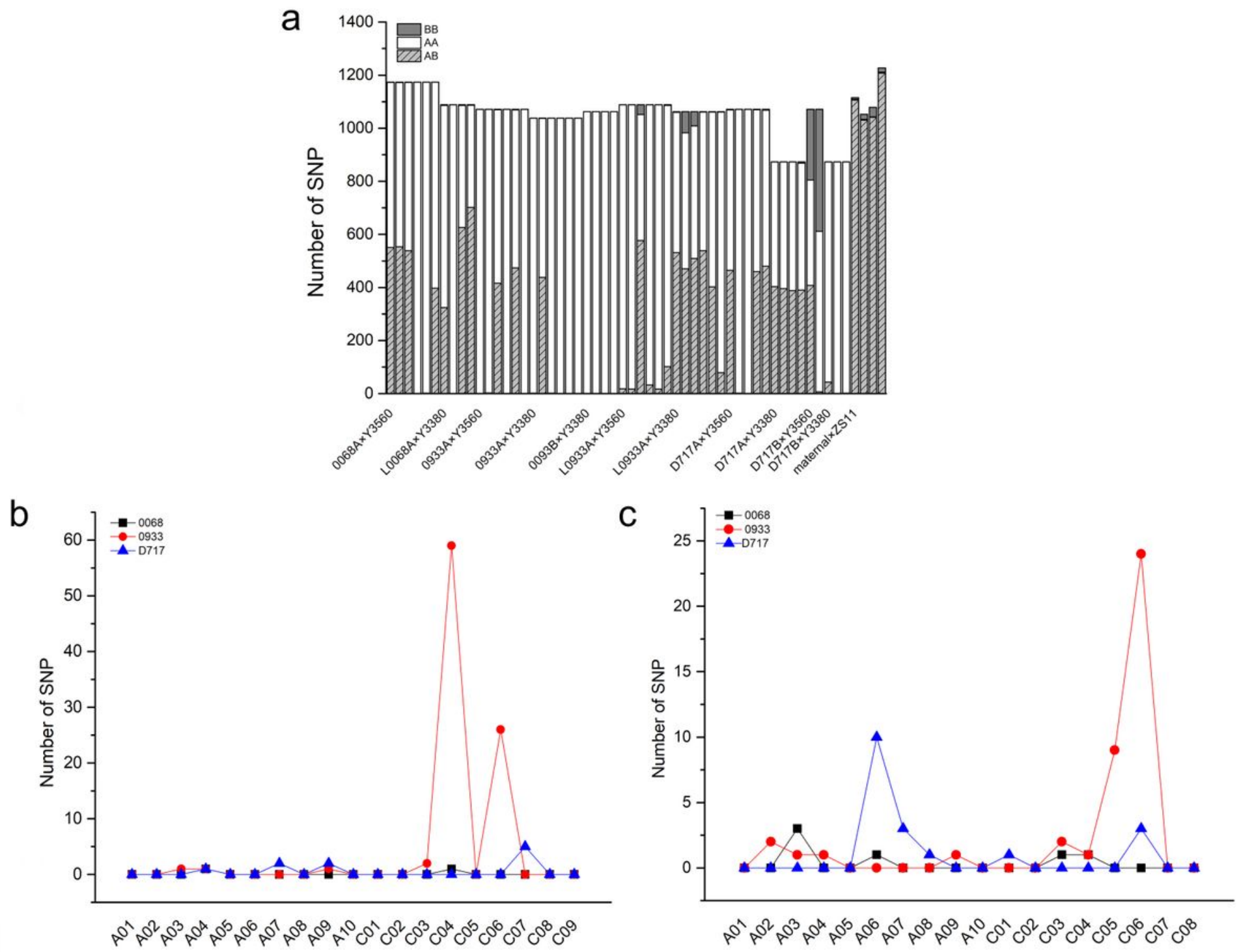

\section{Figure 5}

Induction line induction effect and infiltrated SNP quantity typing statistics. a: Induced line induction effect SNP quantity typing statistics; AA represents the same SNP site as the maternal parent, BB represents the same SNP site as the maternal parent, and AB represents the heterozygous site. b: the DH line is used as the paternal parent, and the distribution of SNP sites infiltrated by the male parent on each chromosome of the induced offspring. c: ZS11 is used as the paternal parent, and the distribution of SNP sites infiltrated by the male parent on each chromosome of the induced offspring.

\section{Supplementary Files}

This is a list of supplementary files associated with this preprint. Click to download.

- Table1.xls

- Additionalfile1.pdf

- Additionalfile10.pdf 
- Additionalfile11.xls

- Additionalfile12.xls

- Additionalfile13.pdf

- Additionalfile2.xls

- Additionalfile3.xls

- Additionalfile4.pdf

- Additionalfile5.pdf

- Additionalfile6.xIsx

- Additionalfile7.xls

- Additionalfile8.xls

- Additionalfile9.pdf 